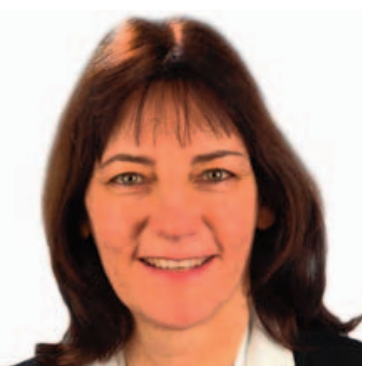

\title{
Allergische Rhinitis als ernstzunehmender Risikofaktor für Asthma - auch bei Rauchern
}

\author{
Alexandra M. Preisser \\ Universitätsklinikum Hamburg-Eppendorf, Zentralinstitut für Arbeitsmedizin und \\ Maritime Medizin, Hamburg
}

Abstractübersetzung aus Verlato G, Nguyen G, Marchetti P, Accordini S, Marcon A, Marconcini R, Bono R, Fois A, Pirina P, de Marco R: Smoking and new-onset asthma in a prospective study on Italian adults. Int Arch Allergy Immunol 2016;170:149-157.

\section{Rauchen und neu auftretendes Asthma in einer prospektiven Studie bei Erwachsenen in Italien}

\section{Schlüsselwörter}

Asthmainzidenz · Rauchverhalten · Allergische Rhinitis . Umgekehrte Kausalität · Italien

\section{Zusammenfassung}

Hintergrund: Zwar sprechen mehrere Studien dafür, dass es eine Ursache-Wirkungs-Beziehung zwischen aktivem Rauchen und neu auftretendem Asthma bei Erwachsenen gibt, nachgewiesen ist dies jedoch bisher nicht.

Ziele: Unser Ziel war die prospektive Untersuchung der Asthmainzidenz als Funktion des Rauchverhaltens in der erwachsenen Bevölkerung Italiens. Methoden: Eine populationsbasierte Kohorte von 5241 Nicht-Asthmatikern wurde im Zeitraum von 1998 bis 2000 in Verona und Sassari in die Studie aufgenommen. Im Zeitraum von 2007 bis 2009 wurde im Rahmen der «GeneEnvironment Interactions in Respiratory Diseases»-Studie erneut Kontakt zu der Kohorte aufgenommen, und 3187 Probanden $(60,8 \%)$ beantworteten einen Screening-Fragebogen zu Rauchverhalten und Atemwegserkrankungen. Die Beziehung zwischen Rauchverhalten und laut Selbstauskunft neu auftretendem Asth- ma (definiert als Asthmaanfälle/Anwendung von Arzneimitteln zur Behandlung von Asthma) wurde anhand eines multivariaten logistischen Modells untersucht.

Ergebnisse: Während des Nachbeobachtungszeitraums traten 145 Neuerkrankungen an Asthma auf, was eine kumulative Inzidenz von 4,6\% ergibt (95\%-KI: 3,9-5,4). Bei der kumulativen Asthmainzidenz bestanden keine signifikanten Unterschiede zwischen Personen, die nie geraucht hatten $(76 / 1,666=4,6 \%)$, solchen, die früher einmal geraucht hatten $(30 / 554=5,4 \%)$ und denen, die aktuell rauchten $(39 / 883=4,4 \%)(p=0,641)$. In einer multivariaten Analyse war allergische Rhinitis der wichtigste Risikofaktor für neu auftretendes Asthma (OR =4,00; 95\%-KI: 3,684,35). Das Risiko für neu auftretendes Asthma war bei Ex-Rauchern im Vergleich zu Personen, die nie geraucht hatten, leicht erhöht $(\mathrm{OR}=1,28 ; 1,09-1,49)$, bei aktuellen Rauchern jedoch nicht $(O R=1,01 ; 0,66-1,53)$. Aktuelles Rauchen war nur dann ein signifikanter Prädiktor, wenn man einen zusammengesetzten Endpunkt aus neu einsetzendem Giemen und neu auftretendem Asthma betrachtete $(\mathrm{OR}=2,03 ; 1,35-3,05)$.

Schlussfolgerungen: In dieser prospektiven Studie ist aktuelles Rauchen kein Risikofaktor für neu auftretendes Asthma, es sei denn, man bezieht neu auftretendes Giemen mit ein. Der erhöhten Asthmainzidenz unter Ex-Rauchern liegt vermutlich eine umgekehrte Kausalität zugrunde.

(c) 2017 S. Karger GmbH, Freiburg

\section{KARGER}

Fax +4976145207 14

information@karger.com www.karger.com (c) 2017 S. Karger GmbH, Freiburg 


\section{Transfer in die Praxis}

\section{Hintergrund}

Der Zusammenhang zwischen inhalativem Tabakrauchen und einem neu aufgetretenen Asthma erscheint auf den ersten Blick recht einleuchtend und selbsterklärend. So zitieren die Autoren auch zunächst 7 Studien, die ein erhöhtes Risiko eines neu aufgetretenen Asthmas bei Rauchern im Vergleich zu Nichtrauchern zeigen. In ihrer Einleitung beschreiben sie jedoch auch 4 Studien, die keinen Unterschied zeigen; eine Untersuchung sah sogar ein geringeres Risiko bei den Rauchern. Rauchen als Schutz vor Asthma? Eine Zusammenfassung der aktueller Studienlage durch das Center for Disease Control and Prevention zeigt, dass ein kausaler Zusammenhang zwischen Rauchen und der Entwicklung eines Asthma als sicher angenommen werden muss, dies betrifft jedoch nicht aktives Rauchen und die Entstehung eines Asthma bronchiale im Erwachsenenalter.

Aus der lesenswerten Literaturübersicht filtern die Autoren wichtige Faktoren, welche diese Studien beeinflussen und zu diskrepanten Ergebnissen führen können:

- Healthy smoker effect: Anfällige Menschen bemerken die schädlichen Auswirkungen des Rauchens früher und beenden das Rauchen eher wieder.

- Die Definition von «Asthma» ist unterschiedlich: In manchen Untersuchungen wird auch wheezing (Giemen) zur Diagnose herangezogen. Falsche Abgrenzungen zwischen Asthma und COPD sind eine weitere Fehlerquelle.

- Risikoerhöhung für Asthma durch Passivrauchen wird unterschätzt: Es werden meist nur Raucher untersucht.

- Studienteilnehmer beenden das Rauchen im Laufe einer Längsschnittuntersuchung: Diese Veränderung muss auch in Folgeerhebungen als eigenständige Variable berücksichtigt werden.

- Sozioökonomischer Status: Es gibt einen Zusammenhang zwischen niedrigerem Sozialstatus und Rauchverhalten, aber auch generell mehr Atemwegserkrankungen.

- Atopie der Raucher: Sie haben ein erhöhtes Asthmarisiko im Vergleich zu nichtrauchenden Atopikern.

Die Autoren haben diese Fragen nun aufgegriffen und drei Längsschnittuntersuchungen (1998-2009) aus dem nördlichen Italien und Sardinien zur Asthmainzidenz überprüft. Es wurden 5241 Personen eingeschlossen, die zu Beginn der Studien nicht an Asthma litten. An den Folgeerhebungen hatten 3187 (40-74\%) Personen teilgenommen.
Zentrale Ergebnisse der genannten Längsschnittstudien

1) Die Entstehung von Asthma wird nicht durch das aktive Rauchen beeinflusst. Dieses Ergebnis war konsistent bei Patienten mit oder ohne allergische Rhinitis und änderte sich nicht nach Bereinigung für den sozioökonomischen Status oder bei der Berücksichtigung, ob die Personen nur bei der Ersterhebung oder auch bei den Folgeerhebungen rauchten.

2) Rauchen zum Zeitpunkt der Ersterhebung war nur signifikanter Risikofaktor, wenn nicht nur die Neuentstehung von Asthma berücksichtigt, sondern auch neu aufgetretenes Giemen als Neuerkrankung gewertet wurde.

3) Ex-Raucher haben ein höheres Risiko für eine Asthmaneuerkrankung. Das erhöhte Risiko schien auf Personen mit asthmaähnlichen Symptomen oder allergischer Rhinitis beschränkt zu sein, war jedoch nicht signifikant.

4) Eine allergische Rhinitis ist der wichtigste Risikofaktor für die Entstehung eines Asthmas.

\section{Fazit für die Praxis}

Aktives Rauchen ist hiernach nur dann ein starker Prädiktor für eine Asthmaneuerkrankung (OR =2,03, 1,35-3,05), wenn die Definition hierfür um Atemwegsbeschwerden in Form von Giemen erweitert wird.

Auch diese Studie konnte also nicht eindeutig klären, ob Rauchen ursächlich für eine Asthmaerkrankung ist. Es zeigte sich jedoch ein anderer wesentlicher Zusammenhang, der mehr Beachtung erfahren sollte. So war die allergische Rhinitis der wichtigste Risikofaktor für die Neuentwicklung von Asthma, verbunden mit einem 4-fachen Anstieg der Asthmaizidenz. Die Autoren betonen, dass sich die meisten Hausärzte der Bedeutung der allergischen Rhinitis als ernstzunehmender Risikofaktor für Asthma nicht bewusst sind.

\section{Disclosure Statement}

Hiermit erkläre ich, dass keine Interessenskonflikte in Bezug auf den vorliegenden Kommentar bestehen.

Kontaktadresse: PD Dr. Alexandra M. Preisser, Universitätsklinikum Hamburg-Eppendorf, Zentralinstitut für Arbeitsmedizin und Maritime Medizin, Seewartenstraße 10, 20459 Hamburg, Deutschland, a.preisser@uke.de 